\title{
Prioritization of Decision Variables for SMBE Cloud based Big Data Solutions Adoption
}

\author{
Somesh Kumar \\ Noida Institute of Engineering \& Technology, \\ Greater Noida
}

\begin{abstract}
Data is becoming the top asset for most organizations and is the key success factor for organizations who are market leaders. The phenomenal growth of Internet and its adoption by consumers has completely changed the market landscape. Consumers are more aware and have various ways of expressing their opinions, thoughts and concerns. Organizations can now no longer ignore these sources of consumer voice and have to be agile enough to use this data to understand the new consumer and his needs. With technological advancement and better business processes, also comes the ability to collect huge data which was never possible before. A typical organization has millions of data records related to functions like Production, Supply Chain, Finance, Customers etc which provide insights into system functionality and maturity.
\end{abstract}

As a bottom line, Organizations have access to Big Data related to its internal processes and external environment which can be used to understand the evolving consumer needs and help these enterprises to move from old age reactive analytics to new proactive and better business interactions and data based business decisions.

Until recently only large organizations had the resources to collect access and crunch this data, providing them a competitive edge over others. But with the maturity of Cloud technology these capabilities are now available to the SMBE sector too. Small and medium business organizations can now use cloud based Big Data tools to move ahead and create a niche for themselves in the market irrespective of cost and technology skill barriers.

This empirical study looks at the Small and Medium Business Enterprise (SMBE) Sector and aims at identifying top five factors / attributes which influence SMBE adoption of Cloud based Big Data Solutions and ranking these factors based on importance.

Data is gathered from SMBEs using various market research techniques including questionnaires and detail interviews. Prioritization of these factors is done using Conjoint Analysis.

The result of this study can be leveraged by Big Data Solution vendors to create cloud offerings specific to SMBE sector.

\section{Keywords}

SMBE, CAGR, CBDS

\section{INTRODUCTION}

Cloud technology is a disruptive technology which is changing the way Business function and use IT resources. Using the metered service model (pay as per use), Cloud technologies have removed the entry barriers for cost, and IT skills for organizations who want to use expensive IT resources. Services are now hosted on Cloud vendor's infrastructure and clients simply use these over a network and are charged only for what they use. Vendors offer services like infrastructure, software, platform, database etc to clients relieving them of the overheads of setting up a complete IT center in house.

With the evolution, maturity and affordability of technology, there was a huge spike in the data generated through different sources. Whether these are consumers talking about products online or data tracked through Retail sales and consumer touch points or through internal end to end production to sale systems; organizations have a lot of data at their disposal. This data is the potential source of Business insights which can help enterprises evaluate their actual performance, understand the changing consumer needs, generate new ideas, better their business processes and identify new markets through innovation.

This huge data is now called Big Data and technologies which allow in gathering, storing, analyzing and presenting this data are called Big Data technologies. IDC defines Big Data solutions [1] as a generation of solutions, tools and architectures designed for extracting value economically from humongous volumes of a wide assortment of data by allowing high velocity capture, discovery and analysis.

In a recent whitepaper published by IDC, it was found that Big Data technology and services market globally will grow at a compound annual growth rate (CAGR) of $39.4 \%$ which is around seven times of the entire Information \& Communication technology (ICT) market. It also states that enterprises which have started to embrace Big Data technology and processes are demonstrating that they can gain competitive advantage not by guesswork but by taking action based on timely, complete, accurate and relevant data insights [5], [7], [9].

Big Data and analytics is an exploding practice today. In the last few years, companies have devoted time and budgets to harness and understand the troves of data around them. This data includes, but is not limited to, social media data (Facebook, Twitter, blogs, Google search), mobile handset usage, digitally enabled processes and transactions, online news, and internal business related administrative records.

Until recently traditional platforms were used for managing Big Data but now specialized tools and techniques have emerged which specifically aim at Big Data and its efficient and real time analysis through new algorithms and paradigms. These technologies require expensive hardware installation and complex frameworks and could only be afforded by organizations with deep pockets and skilled resources. This acted like an entry barrier for smaller organizations that could greatly benefit from Big Data but could not afford the upfront investments.

Emergence and maturity of Cloud technologies and new 
offerings around Big Data tools has changed the game field. Now Cloud Based Big Data Solutions (CBDS) or Big Data as a service is the new trend which is leveling the market landscape and allowing all organizations to use these services at hardly any cost as compared to the initial infrastructure setup required before.

Cloud has made redundant the benefits of size, scale and scope previously held by a few large enterprises and opened up a new front for smaller companies to leverage the benefits of Big Data technologies. It has drastically cut down the investment arbitrage for new and small players.

\section{CBDS AND SMBE SECTOR}

Small and Medium Enterprises have grown exponentially with the spurt in technology adoption and new consumer mindset. Big players in all sectors are being threatened by the small and medium business strategies. The government has also come up with various policies to help the SMBE sector grow. Findings in a recent study the SMB Chamber of Commerce and Ministry of Micro, Small \& Medium Enterprises, there are around 48 million small and medium business enterprises in India, contributing $45 \%$ of industrial output, $40 \%$ of total exports and 1.3 million yearly jobs [1].

In a recent survey by GreyHound Knowledge group [2] SMBEs in India are facing growth challenges out of which the biggest are unavailability of affordable technology, lack of infrastructure and availability of Finance. The affordability of Cloud based technology has come as a relief for SMBEs. SMBEs have realized the importance of adopting technology in business processes and decision making and now with the possibility of having Big data technology within reach through Cloud vendors, SMBEs increasingly are looking forward to integrating their business intelligence with these CBDS to leverage the strength information brings.

This study concentrates on three SMBE sectors.

1. Telecom Sector: Based on a report by Analysys Mason, it is forecasted that worldwide revenue from SMBEs for Information and Communication technology (ICT) sector will grow from $\$ 203$ billion in 2012 to $\$ 226$ billion in 2017. A lot of SMBEs are growing in this sector and big players are collaborating with these SMBEs. The telecom market in the Indian subcontinent is around $\$ 800$ million out of which SMBEs contribute to almost one fourth of the market share (more than $\$ 200$ million).

2. Retail: Small and Medium Business enterprises in Retail Sector are estimated to generate revenue of more than $\$ 450$ Billion, and comprise of almost $95 \%$ of the overall Indian Retail sector. This sector is growing exponentially with better practices and government support. Also FDI is playing a major role in transforming the market landscape.

3. Health: Based on the research done by the rating agency Fitch, the Health sector in India is estimated to grow to \$ 100 billion by 2015 , with a $20 \%$ yearly growth[8]. With changing lifestyles, increase in medical tourism and an increasing need for quality and specialized health facilities, this industry is expected to jump to $\$ 280$ billion by 2020 .

SMBEs have yet to utilize the full potential of the Cloud technology and specially Big Data tools. The availability of huge transaction and customer data is now making SMBEs take a serious look at these CBDS technologies for business edge and growth. The cost advantages which Cloud brings by drastically reducing initial CAPEX and OPEX has made expensive infrastructure and solutions affordable and lucrative to these industries [10-12]. SMBEs which are mostly entrepreneurial or family based businesses and used to base business decisions mostly on personal networks and dated concepts and knowledge can now avail deep data insights which were not possible earlier leading to rapid time to market, better consumer understanding, accurate forecasting, sleek supply chains and better cash flows.

Despite the known advantages, the SMBE sector faces a lot of challenges in adopting cloud technologies [3] and Big Data frameworks due to various internal and external constraints like:

- Absence of clear technology driven business value chain benefits

- Vague understanding of technology and its integration in existing business processes

- $\quad$ Significant upfront technology investment

- $\quad$ Shortage of skilled personnel

In a previous research by the authors, ten satisfiers and ten dissatisfiers were identified based on interviews with various industry experts and SMBE leaders. These variables were found to influence the decision of cloud based big data adoption by small and medium business enterprises [4]

\section{SATISFIERS}

\subsection{Cost Effective:}

Clients perceive benefits of upfront cost saving by adopting cloud solutions. Immediate CAPAX is saved on infrastructure costs and OPEX is saved on usage since Cloud models are based on utility payment models. The client only pays for the services it consumes.

Cloud vendors also provide different kinds of payment methods like one time pay, pay per use etc.

\subsection{Innovation}

Big Data solutions allow enterprises to understand the real time changes in the business landscape and hence be nimble enough to look for new business opportunities or identify better business process or Innovate. Innovation sprouts from enterprises which are business focused and have business processes based on insights from real time data. Big Data gives deep and real insights on what is going on and what is needed.

\subsection{Location}

Cloud services allow even the remotest of SMBEs to connect to the vendor services and avail access CBDS. This has been possible only because of successful deployment of Big Data tools on Cloud. Location and large distances are no longer a limitation for enterprises.

\subsection{Convenience}

Cloud based Big Data tools have an inherent advantage of providing continuous and universal access to consumers. Data and tools can be accessed from anywhere, anytime on any device.

\subsection{Speed \& Scales}

SMBEs have the advantage of scaling up or down the services it avails based on business needs and pay only for what they consume saving immediate OPEX. Also the time lag between identifying a business need for Big Data and getting access to 
a Big Data tool is minimal as Cloud services can be made available almost immediately.

\subsection{Multiple Users, Mobility and Device independence}

Cloud enables offerings to be device independent and be accessed by multiple users on the go anywhere. These services can range from a salesman entering data on a handheld device on the shop-floor to the senior managers sitting at remote locations making real-time decisions based on the insights generated by data analytics over cloud.

\subsection{Flexibility}

CBDS allow enterprises to pick and choose services from a set of offerings provided by the vendor, based on current business maturity and need. Alternately in a non-cloud platform, complete platform needs to be deployed for using only a small part of it.

\subsection{Unlimited Storage Space and Computing power}

Big Data analytics requires high storage and computing power for storing and analyzing data. Cloud platforms provide this computing power and storage which can be scaled based on needs.

\subsection{Customize Settings}

Enterprises can dictate the vendors to tweak the services based on their unique business requirements. Cloud vendors allow consumers to cherry-pick and tailor what they need and also pay only for what they use.

\subsection{Software and Hardware Updates}

The Cloud consumer is free of any overheads regarding maintaining the hardware or ensuring software updates and installing patches. This is the responsibility of the cloud vendor leaving the consumer to focus on its core business.

\section{DISSATISFIERS}

\subsection{Data Security}

Data is one of the biggest assets an organization can have and data security is a major issue. SMBEs have concerns in putting their critical and sometimes confidential data on cloud which may eventually reside on servers in remote locations under a risk of malicious access over the network.

\subsection{Data Privacy}

Since data is now available on the network, there is a threat to the data which comes from internal employees and the way they access the tools. No matter how strong the vendor secures the data, a simple password misplaced by an employee can render all security useless.

\subsection{Data Availability:}

The cloud is dependent on a constant availability of the network. There is an inherent fear of not getting access to the cloud in case the network goes down due to uncontrollable reasons leaving the business separated from the data it needs.

\subsection{Vendor Lock In}

Organization data may run into terabytes of information over time. This creates a situation where the consumer of cloud services becomes dependent on the cloud vendor simply because the cost of moving to a different vendor becomes more than the cost to continue with the current vendor creating a vendor lock in situation. This lock in may be because of many reasons like incompatibility with the underlying technology, data structures, application restrictions to user getting comfortable with the vendor framework.

\subsection{System and Process compatibility}

SMBEs usually have an existing set of tools, either in-house or small third party, which are critical to the functioning of the business. Any decision to move to the CBDS may result in compatibility issues between these solutions and the cloud offerings. There can also be a case of conflicts over the way business functions with the way the cloud vendor functions.

\subsection{Location of Data}

Cloud benefits include providing solutions to anywhere in the globe, but this also means that organization data could be stored anywhere. This could result in export control (ITAR / EAR) issues for research data. Enterprises may also be uncomfortable in storing data in enemy countries or countries with security and accessibility laws different from their own.

\subsection{Duration of Services}

Vendor service and the duration of the services offered have an important impact on the decision of partnering with a vendor. SMB organizations may be looking at a small term engagement and a cloud vendor which provides flexible options suiting SMBEs preferences and business needs will be rated higher than those who don't provide flexible service durations.

\subsection{Responsibility for End Users}

SMBEs need to be educated on the service terms, policies and scope of the services offered. Typically SMB enterprises do not have the technical expertise to fully understand the terms and vendor's Acceptable use Policy (AUP) on the big data services and hence expectation mismatch could lead to failed implementations of cloud.

\subsection{Ownership of Data}

SMBEs may be hesitant to move their critical data on vendor cloud due to ownership questions. Since the cloud is not in their control, a debate arises that who will own and be eventually responsible for the data and consequently the insights generated from this data since the vendor owns the infrastructure on which the data is stored and the tools which helped generate the insights. This brings the question of whether the cloud vendor can use Intellectual Property Rights (IPR) and use client data for personal use simply by the virtue of transactions.

\subsection{Bandwidth, quality of service and data limits}

Since the Cloud is completely network dependent, there could be issues of outages due to network failures, slow connection speeds etc. resulting in unavailability of data and services to the client which may have a direct dollar impact.

\section{PROBLEM FORMULATION AND RESEARCH METHODOLOGY}

As per a study by The Economist in 2013 named "The hype and the hope"[3], it was found that firms in Asia Pacific which had implemented Big Data tools and solution reported limited success in implementation even when they did report that they had a huge and increasing opportunity to utilize Big Data. Two of the top reasons identified were:

- Lack of suitable software to suit the business requirement

- Lack of necessary skills to implement Big Data 
technology in the organization

This study clearly shows that understanding the correct technology required for the business and the right skillset to implement it has a major impact on Big Data implementation for an organization. For SMBEs are still worried and conscious of moving to Cloud based Big Data technology because of a vague understanding of the technology and its fitment. Cloud vendors need to be cognizant of the priorities of the SMBEs and the right tools and frameworks which suit these sectors and create offerings based on these. This paper takes a step in this direction.

The authors in a previous study identified top ten satisfiers and dissatisfiers for CBDS adoption by SMBE sector. These were grouped into four Gain drivers and Risk drivers. Interviews with industry leaders and sector experts were used to condense these four gains from the satisfiers and four risks from the dissatisfiers which were related to four independent and unrelated gain and risk drivers.

The satisfiers and dissatisfiers which integrate into each factor are tabulated and shown in Table 1:

Table 1 four posited GAINS of CBDS

\begin{tabular}{|l|l|}
\hline \multicolumn{1}{|c|}{ Gain 01 } & \multicolumn{1}{|c|}{ Gain 02 } \\
\hline Cost Saving & Better Prediction \\
- $\quad$ Cost Effective & $\bullet \quad$ Innovation \\
- Software and hardware & $\bullet \quad$ Convenience \\
- $\quad \begin{array}{l}\text { Clexibility } \\
\text { - }\end{array}$ & \\
Customize Settings & Speed and Scales \\
\hline
\end{tabular}

\begin{tabular}{|c|l|}
\hline \multicolumn{1}{|c|}{ Gain 03 } & \multicolumn{2}{|c|}{ Gain 04 } \\
\hline Consumer Insights & $\begin{array}{l}\text { Accurate and Real Time } \\
\text { Data }\end{array}$ \\
& $\bullet \quad$ Location \\
- $\begin{array}{l}\text { Unlimited Storage } \\
\text { Space and Computing } \\
\text { Power }\end{array}$ & $\begin{array}{l}\text { Multiple } \\
\text { Mobility and Device } \\
\text { Independence }\end{array}$ \\
\hline
\end{tabular}

\begin{tabular}{|l|ll|}
\hline No Gain & \multicolumn{2}{|l|}{ Efficiency Risk } \\
$\begin{array}{l}\text { Risk of no gain from } \\
\text { CBDS adoption or } \\
\text { only Short term gain. }\end{array}$ & $\bullet$ & Data Availability \\
& Speed and Scales \\
& $\begin{array}{l}\text { Bandwidth, quality of service } \\
\text { and data limits }\end{array}$ \\
\hline
\end{tabular}

Table2 four posited RISKS of CBDS

\begin{tabular}{|c|c|}
\hline Risk 01 & Risk 02 \\
\hline $\begin{array}{l}\text { Vendor Risk } \\
\text { - Vendor Lock in } \\
\text { - } \quad \text { System and Process } \\
\text { Capability } \\
\text { - Duration of Services } \\
\text { - } \quad \text { Responsibility of End } \\
\text { users } \\
\text { - Ownership of Data }\end{array}$ & $\begin{array}{l}\text { Security Risk } \\
\text { - } \quad \text { Data Security } \\
\text { - } \quad \text { Data Privacy } \\
\text { - } \quad \text { Location of Data }\end{array}$ \\
\hline
\end{tabular}

A sample space of 116 SMBE experts was chosen from a diaspora of Telecom, Retail and Health SMB Enterprises. These experts were divided between Telecom sector (34 respondents), Health sector (39 respondents) and Retail sector (43 respondents).

Most of the respondent population had not yet adopted CBDS significantly and hence the responses are purely based on the respondent perceptions of the gains and risks CBDS brings to their respective sectors and organizations.

\subsection{Method}

Big Data technology on Cloud not only enables SMBEs to save upfront investments as CAPEX and allocate capital to core Business functions, but also helps them in gaining market insights, perform better PR activities, faster time to market, uncover trends, accurate forecasting, streamline processes etc. The utility based payment model (pay as per use) of cloud computing technology is favorable to SMBEs which saves a lot of OPEX on skill and infrastructure maintenance. Thus cost saving is one of the first gain driver. Big Data technology also helps enterprises in understanding trends and forecasting. This is the main advantage of Big Data mining which helps uncover variables and trends which help organizations understand a behavior and predict what can happen. SMBEs can then plan product launches or better system processes based on these predictions. Similarly Vendor risk and security risk which are inherent to Cloud technology are two of the risk variables. SMBEs are wary of putting their data in external servers where a risk of data theft or loss is a possibility.

This research paper aims at identifying the preferences of SMB Enterprises on the decision variables involved in adopting Cloud Based Big Data Solutions in their business. These are anticipated or perceived preferences and not based on ex-post adoption feedback. We identify using conjoint analysis the relative priority of gain and risk variables which the SMBE sample space perceives is important to their sectors. This is done for Telecom, Health and Retail sectors separately.

- The sample space for the research consists of 116 SMBE leaders and industry/sector experts.

- These are from the Telecom, Retail and Health sectors.

- Telecom sector: 34 respondents

- Health sector: 39 respondents

- Retail sector : 43 respondents 
- This sample of respondents has not yet adopted CBDS significantly in their organizations and hence their responses are purely based on ex-ante perceptions and not on post adoption feedback.

- Conjoint Analysis has been used to rank the professed priority of drivers and inhibitors for CBDS adoption by SMBEs.

- Data Collection: Data was collected through an online questionnaire which was released to SMBEs all over India. The respondents were guided over audio and skype calls (wherever face to face meeting was not possible) to the context of the questionnaire.

\section{- Questionnaire:}

- Respondent details: We gather the name and age of the respondent. Apart from basic response identification, these fields may also help us understand the difference in perceptions of the young or mature respondents.

- Industry Sector: This field helps us understand the sector to which the organization belongs to. We also capture the name of the organization so that in case more details regarding the firm can be recovered if required.

- Drivers favoring CBDS Adoption: Following are the gain drivers.

- Cost Saving: Perceived advantages of revenue increase and cost reduction. CAPEX gains due to cut in upfront investments in architecture and OPEX gains due to the utility model of payment inherent to cloud based services. It also includes no cost on software and hardware updates (as this falls in the vendor domain) and the services being offered in a-la-carte fashion allowing SMBEs to choose services they need and ignore others.

- Better Prediction: Advantages of insights from Big Data which help uncover trends and variables which help SMBEs in building improved marketing strategy, launch of products and services, tweak and better business processes and help innovate and build next generation offerings. All this based on the convenience of having Big Data technology available over cloud for different departments with the data view they need for forecasting and prediction.

- Consumer Insights: Big Data helps enterprises understand the voice of the consumer and hence brings them closer to the customer needs. Enterprises can then move from creating products and services designed for mass to personalized solutions for individual consumer unique to his/her needs. The advantage cloud offers is unlimited storage and raw computing power which enables organizations to store collect data from all relevant resources and then churn this data to generate real time insights.

- Accurate and Real time Data: A key driver for successful business decision making is the availability of right information in the right way to the right people. Cloud and Big Data technology allow huge data to be processed real time and made available anytime to the management in a location and device independent way.

- Drivers deterring CBDS adoption:

- Vendor Risk: Risks associated with the specific vendor with which the SMBE deals with. This could be the risk of technology lock up wherein the data once stored in the vendor infrastructure is difficult to migrate to other vendor in case the SMBE wishes to change the vendor, to Vendor Lock in incompatibilities in the system and processes of the vendor. Also post implementation support, responsibilities of the customer, duration of services, ownership of data etc create a part of vendor risk.

- Security Risk: Risks affecting organization data security, privacy, integrity, access. Data is a key winning asset for any organization and security of that data is a critical concern.

- Efficiency Risk: Due to the inherent dependency of Cloud technologies on network, data availability and speed depend on the available network strength. Hence bandwidth, quality of data, download and upload speeds etc are a concern for efficient utilization of CBDS.

- No Gain Risk: The fear that adopting CBDS will lead to no long term tangible gain for the organization. All effort and transformation will bring only marginal or no gain to the business process and decision making.

- The Gain drivers and the Risk drivers are grouped in combination of two's and the respondents were requested to rank these six combinations on a scale of 1-100 such that the total sum of ranks has to be equal to 100 . Hence respondents were forced to rate their perceptions by allocating 100 points to each of the six combinations.

- This questionnaire was sent to SMBEs in three sectors: Telecom, Retail and Health.

- Post data gathering, conjoint analysis was run on the survey results to identify which Gain and Risk drivers were important to the SMBE for these three sectors.

\subsection{Analysis}

The aim of this research is to identify a ranked associativeness of the four decision variables on the decision by Small and Medium business enterprises for Cloud based Big Data Solution adoption. All the gain are important to the SMBE sector but ranking them helps understand the subtle differences between the three sectors on their perceptions about CBDS and priorities[6]. Tradeoff between these drivers helps understand patterns and eventually the fundamental working of these sectors.

Telecom Sector:

The ranking of the four gain drivers for telecom SMBE is given in Figure 1 below. It can be clearly seen in the figure that Cost Saving including both CAPEX and OPEX and 
availability of accurate and real time data take $63 \%$ of the gain perception spectrum of the SMBE in Telecom sectors. Better prediction and Consumer Insights take a lower ranking.

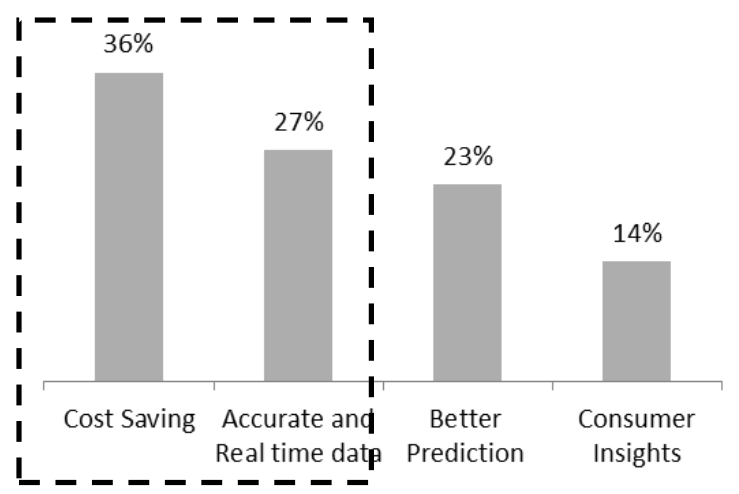

Figure 1: Gain Drivers for Telecom SMBE Sector

The ranking of the risk perceptors for telecom sectors can be seen in the Figure 2 below. Vendor risk seems to be the top concern for telecom sector. This is evident of the huge data that telecom companies capture leading to vendor locking risk perception. Efficiency is also a major concern. A lot of data in this sector is captured real time and availability of this data for analysis is key to business success. An interesting fact captured is that Security risk seems to be of less concern to Telecom SMBE sector. This could be attributed to the understanding that SMBEs do not have access to the confidential consumer data or such data is not planned to be moved on Cloud for analysis due to strong industry governance and laws.

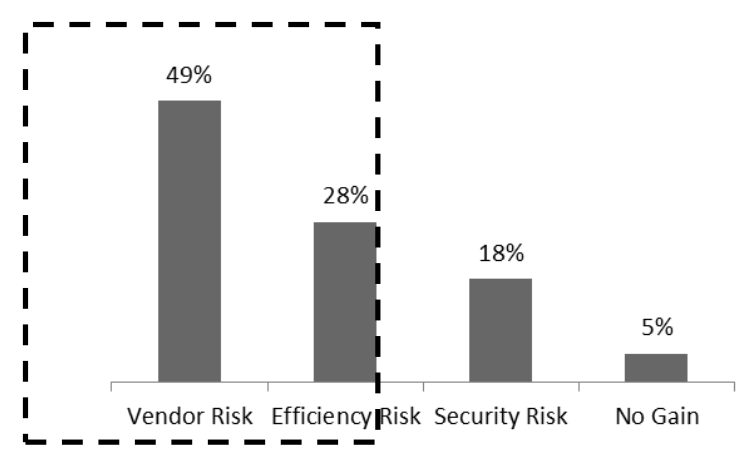

Figure 2: Risk Drivers for Telecom SMBE Sector

\section{Health Sector:}

The ranking of the gain drivers is given below in Figure 3 . Both the Cost Saving and Better prediction gain drivers take almost $70 \%$ of the perception spectrum. Consumer insights and Accurate and Real time data seem to be at a lower priority for Health sector. Health SMBEs usually have tight budgets and hence Cost saving seems to be the top logical gain driver. Further predicting consumer patterns seems to drive the CBDS adoption by Health SMBEs

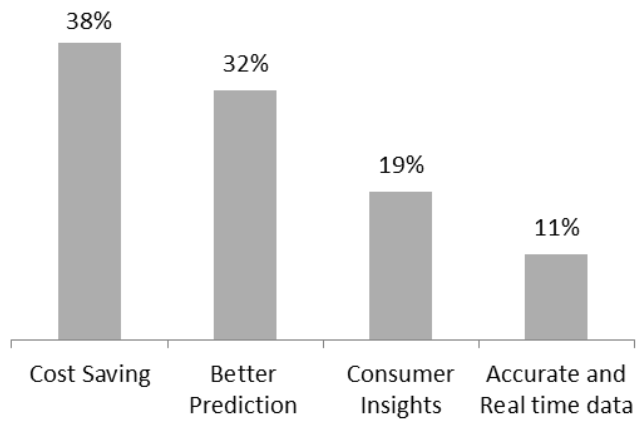

Figure 3: Gain Drivers for Health SMBE Sector

The risk driver ranking for Health sector is given below in Figure 4. A clear priority to Security and Efficiency risk is given which amounts to total $74 \%$. Health records are confidential and SMBEs understand the risk of losing this data. Vendor risk and No Gain are of least priority.

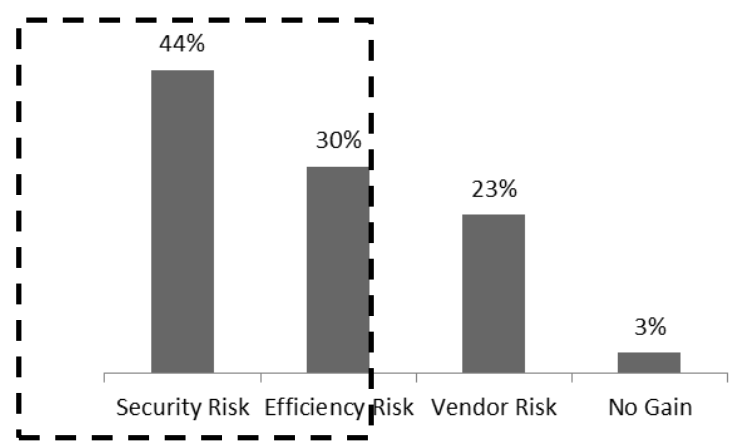

Figure 4: Risk Drivers for Health SMBE Sector

Retail Sector:

The ranking for Retail sector gain perceptors is given in the Figure 5 below. It can be seen that Cost saving is the highest priority with $39 \%$ but Accurate and Real time data closely follows with $31 \%$ both making the $70 \%$ of Gain perception for Health SMBE sector. As a cash conscious sector, Cost saving is the logical top priority. Success in Retail sector is based on carefully monitored supply chain and availability and flow of real time accurate data between the various processes in the product life cycle. Prediction is also important by compared to availability of accurate data ranks less.

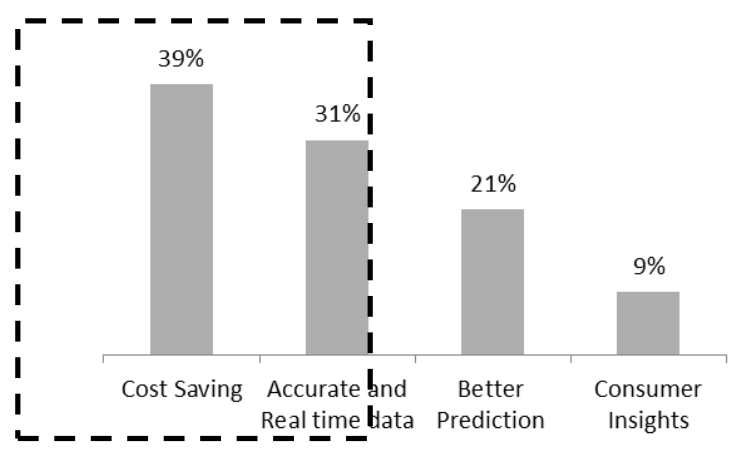

Figure 5: Gain Drivers for Retail SMBE Sector

The risk driver ranking for the retail sector is given in the Figure 6 below. Data security risk is the predominant risk for the Retail sector followed closely by Efficiency Risk. Both contribute to $70 \%$ of the risk perception spectrum. Vendor risk seems to be low priority with $19 \%$ of spectrum. Retail 
industry deals with financial information like credit card details leading to security concerns over data. Also in the extremely competitive Retail sector it is important to get real time information on sales and customers making efficient risk as second priority for retails enterprises.

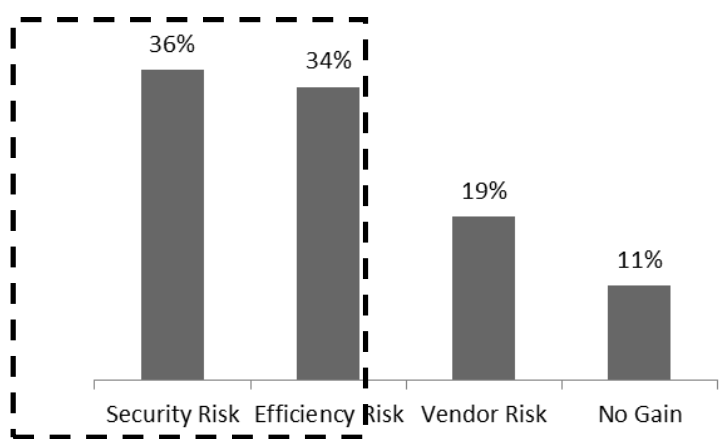

Figure 6: Risk Drivers for Retail SMBE Sector

Overall for all three sectors it if found that Cost Savings is the top gain driver for SMBEs. Cloud platforms and the Utility based payment methods has really attracted the SMBE sector which now has access to all the latest technology infrastructure and tools without the overheads of maintaining and upgrading these setups. The "How-to" of the process is effectively out-sourced to the cloud vendors thereby leaving the business to focus on "What-to" of their core business domain. The SMB enterprises which are usually strapped for cash focus on Cost saving as a major benefit. Coming to the next gain driver, the availability of accurate and real time data, except the Health sector, both the Telecom and Retail sectors view it as priority. This can be attributed to the fact that Telecom and Retail sectors need real time or near real time data from across its systems for business processes, for Telecom it could be network optimization or actual usage of services whereas for Retail it could be stock or product location control, accurate and real time information is key in success. Better prediction is the third driver which the Health Sector marks at a higher priority than the other two sectors. This may be due to real time customized package offers to walk in customers in health clinics based on predictive analysis of past medical tests and possible ailments.

Moving on to the risks, Security risk dominates the risk perception spectrum among Health and Retail sectors. This could be due to the sensitive financial and customer information these sectors have to handle. Telecom on the other hand does not consider this as a top risk may be due to already heavy regulations around the consumer data not allowing SMBEs to migrate it over cloud. Efficiency risk has been ranked as second risk driver for all the three sectors. The decision to move on Cloud brings with it the risk of complete dependency on the available network. In case the network goes down or bandwidth is impacted, this could adversely affect the ongoing systems delivering a blow to the business. Hence the perceived problems with data transfer between the vendor and client to latency issues affect the SMBE decision to adopting CBDS in their organizations. Telecom sector ranks Vendor Risk as a top driver whereas the other two do not. Telecom SMBEs work with huge data and the risk of getting stuck with all this data overtime with a single vendor is real. Hence choosing a vendor has to be done taking in consideration a lot of vendor specifications. The No-Gain driver takes the least priority in the SMBE mind set. This clearly shows that all sectors see value in moving to Cloud platforms hosting Big Data technology. This perception is the reason we see mass SMBE migration to Cloud.

The aim of this research study is to aid cloud vendors in planning new offerings for the SMBE sector based on the right tools, price points, security processes and service levels. But the authors also understand that SMBE sector need to see more successes CBDS implementation, more case studies, proven technology frameworks and tool recommendations to feel comfortable and move to adopting Big Data on Cloud with confidence. Our study will pave way to a clear the fog of qualm surrounding the cloud offerings for the SMBE market.

\section{RESULTS}

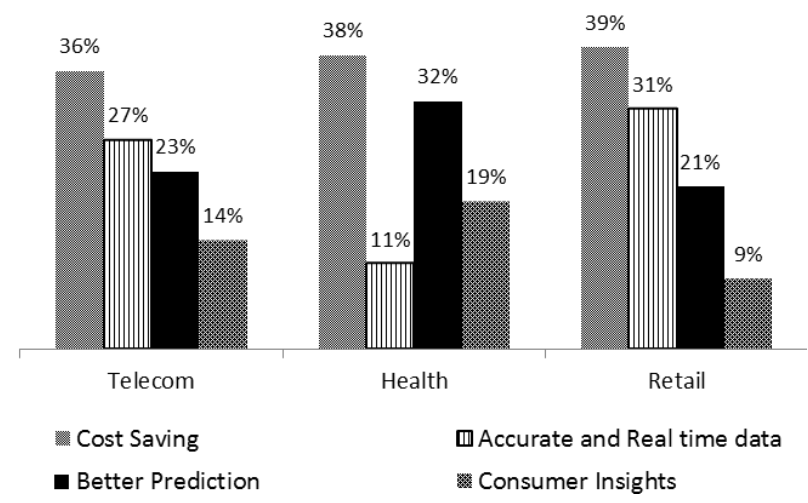

Figure 7: Gain Drivers for all three SMBE Sectors

1. As seen in Figure 7 above which shows the Gain drivers for all SMBE sectors, Cost savings are the top ranked gain drivers for all three sectors. All three sectors rate it close to $40 \%$ of the mindset spectrum

2. Since SMBEs are usually strapped for cash, Cost savings is the most attractive driver for CBDS adoption. Specifically the CAPEX savings inherent to Cloud, saving SMBEs from making huge upfront infrastructure investments before actually seeing the technology gains

3. Accurate and real time data is the next top gain driver for Telecom and Retail since both sectors rely decisions taken real time like network optimization and supply chain tracking

4. Better prediction capabilities of Big Data technology is the third driver which is rated higher in Health sector than other two sectors

5. Consumer Insights has been ranked the least important as compared to the other three drivers by all three sectors. This may be due to the perceived lack of maturity in Big Data technology for SMBE sector in generating meaningful consumer insights to actually show a dollar impact on the revenue

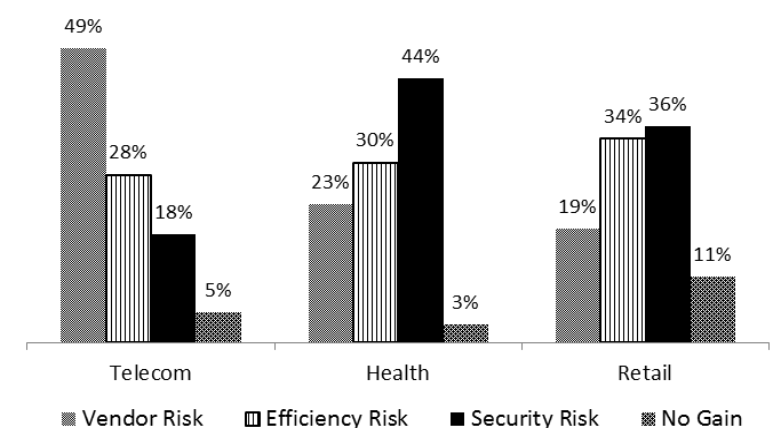

Figure 8: Risk Drivers for all three SMBE Sectors 
1. All Risk drivers for three SMBE sectors is shown above in Figure 8. Vendor risk is the top rated risk in SMBE adoption of CBDS in Telecom sector. This encompasses

a. Vendor Lock in due to technology binding

b. Service durations and the available payment models

c. Client responsibilities vs Vendor responsibilities

d. Data ownership issues

2. Vendor risk is not the top risk as perceived by Retail and Health sectors.

3. Efficiency related risks take the second position in SMBE mindset for all three sectors.

4. Security risk is highest rated risk for Health and Retail sectors but not so much for Telecom.

5. Telecom is a heavily regulated sector and hence secure data is never planned to be moved to cloud. Health and Retail sector on the other hand have to look at data security issues before migrating.

6. SMBE sector has an understanding of the value CBDS could bring to the organizations and hence all three sectors rate No Gain as the least concern.

\section{CONCLUSION}

All organizations are experiencing a boom in the available data from various sources including external sources like social media platforms, online customer feedbacks, product purchase patterns to internal sources like system logs, data generated during production etc. With the onset of the Internet-of-Things (IOT) we are now looking at huge waves of data generated through systems and devices. All this data is growing exponentially leading to a situation where existing systems are overwhelmed or simply unable to store and analyze this data. On the other hand this data beings with it information, patterns and insights which could mean a game changer for the SMB enterprises. Organizations now are moving away from reactive analytics to real time proactive customer touch points and interactions. Insights generated through this Big Data lead to competitive advantages and optimal returns. Cloud vendors now bring Big Data technology to their platforms leading to availability of these technologies to the SMBE sector bringing with it the inherent advantages of Cloud like Utility model of payment to almost zero CAPEX investments.

Through this paper we have looked at the perceived gains and risks of the SMBEs for Telecom, Retail and Health sectors. This helps cloud vendors in understanding the subtle differences in the needs of these sectors and create offerings targeting them.

\section{FUTURE DIRECTIONS OF RESEARCH}

With a plethora of tools and technologies in the Big Data space available today, it is difficult for SMBEs to understand which suits their needs and priorities. Based on the findings of this paper, a further study of these tools can be done. They may be ranked based on the gain and risk drivers and specific offerings may be created by Cloud vendors for these sectors.

\section{REFERENCES}

[1] http://articles.economictimes.indiatimes.com/2013-0609/news/39834857_1_smes-workforce-small-andmedium-enterprises

[2] http://firstbiz.firstpost.com/sme-report/pdf/AnalysingIndian-SME-perceptions-around-Union-Budget-2014 15_Final-new.pdf

[3] https://www.hds.com/assets/pdf/the-hype-and-the-hopesummary.pdf

[4] Prabhjot Singh Lamba et All, Decision Variables for SMBE Adoption of Cloud Based Big Data Solutions, International Journal of Advanced Trends in Computer Applications, 2395-3519

[5] E llaway RH, Pusic MV, Galbraith RM, Cameron T (2014) Developing the role of big data and analytics in health professional education. Med Teach 36(3):216-222

[6] E llaway RH, Pusic MV, Galbraith RM, Cameron T (2014) Developing the role of big data and analytics in health professional education. Med Teach 36(3):216-222

[7] Big data: The next frontier for innovation, competition, and productivity. James Manyika, Michael Chui, Brad Brown, Jacques Bughin, Richard Dobbs, Charles Roxburgh, and Angela Hung Byers. McKinsey Global Institute. May 2011.

[8] Seebode C, Ort M, Regenbrecht C, Peuker M (2013) BIG DATA infrastructures for pharmaceutical research. IEEE International Conference on Big Data, California.

[9] Bellini P, di Claudio M, Nesi P, Rauch N (2013) Tassonomy and review of Big data solutions navigation. In: Big Data Computing. Chapman and Hall/CRC, Boca Raton. p 57

[10] http://www.ey.com/Publication/vwLUAssets/EYSMAC-the-next-growth-driver-for-SMEs-inIndia/\$FILE/EY-SMAC-the-next-growth-driver-forSMEs-in-India.pdf

[11] Amit Singh Sisodiya, "SMEs in India - Future Perfect", Global CEO, April 2006, The ICFAI University Press

[12] http://www.deloitte.com/assets/DcomIndia/Local\%20Ass ets/Documents/Thoughtware/TMT\%20India\%20Predicti ons\%202012.pdf 